\title{
N-myc downstream-regulated gene 1/Cap43 expression promotes cell differentiation of human osteosarcoma cells
}

\author{
TORU MATSUGAKI ${ }^{1}$, MICHIHISA ZENMYO ${ }^{1,3}$, KOJI HIRAOKA ${ }^{1,2}$, NOBUHIRO FUKUSHIMA ${ }^{1}$, \\ TAKANORI SHODA $^{1,2}$, SETSURO KOMIYA $^{3}$, MAYUMI ONO ${ }^{4}$, MICHIHIKO KUWANO ${ }^{5}$ and KENSEI NAGATA ${ }^{1,2}$ \\ ${ }^{1}$ Department of Orthopaedic Surgery, ${ }^{2}$ Research Center of Innovative Cancer Therapy, Kurume University \\ School of Medicine, Kurume 830-0011; ${ }^{3}$ Department of Orthopaedic Surgery, Graduate School of Medical \\ and Dental Sciences, Kagoshima University, Kagoshima $890-8520 ;{ }^{4}$ Department of Pharmaceutical \\ Oncology, Graduate School of Pharmaceutical Sciences, ${ }^{5}$ Innovative Center for Medical \\ Redox Navigation, Kyushu University, Fukuoka 812-8582, Japan
}

Received May 6, 2010; Accepted June 25, 2010

DOI: 10.3892/or_00000913

\begin{abstract}
The $\mathrm{N}$-myc downstream regulated gene 1 (NDRG1)/Cap43 is closely associated with cell differentiation, and its expression is induced by hypoxia and increasing intracellular calcium levels. Whether the NDRG1/ Cap43 expression in cancer cells is a predictive marker of good or poor prognosis in patients, depends upon tumor types and differentiation status. In this study, we examined whether the NDRG1/Cap43 expression was involved in the differentiation of osteosarcoma cells, using three osteosarcoma cell lines, MG63, U2OS and SaOS2. The NDRG1/Cap43 expression in MG63 and U2OS was significantly enhanced by vitamin $\mathrm{D}_{3}$, which also induced the production of osteocalcin, a differentiation marker of osteoblasts. The knockdown of NDRG1/Cap43 using small interfering RNA also suppressed the production of osteocalcin and enhanced cell proliferation, accompanied by the suppression of p21 expression. Furthermore, the acquired invasiveness of osteosarcoma cells during the invasion in Matrigel resulted in the decreased expression of NDRG1/Cap43. On the basis of these results, our proposed role for NDRG1/Cap43 would be in the capacity of differentiation and invasion in osteosarcoma cells.
\end{abstract}

\section{Introduction}

The N-myc downstream regulated gene 1 (NDRG1)/Cap43 gene, also known as Ndrg-1 (1), Drg-1 (2), RTP (3), and RIT42 (4), was isolated using the differential display technique, as was done previously with the gene induced by nickel

Correspondence to: Dr Michihisa Zenmyo, Department of Orthopaedic Surgery, Graduate School of Medical and Dental Sciences, Kagoshima University, 8-35-1 Sakuragaoka, Kagoshima 890-8520, Japan

E-mail: kumikohe@m2.kufm.kagoshima-u.ac.jp

Key words: N-myc downstream regulated gene 1, Cap43, osteosarcoma, small interfering RNA compounds in human bronchoalveolar epithelial cells (5). This gene expresses a $3.0-\mathrm{kb}$ mRNA encoding a $43-\mathrm{kDa}$ protein. The NDRG1/Cap43 protein possesses three unique 10-amino-acid tandem repeats at the $\mathrm{COOH}$-terminal end. Analysis of the amino acid sequence predicted that the protein contains seven or more phosphorylation sites and that it is phosphorylated by protein kinase A (6). Many factors and chemical agents control the expression of NDRG1/ Cap43. The expression of NDRG1/Cap43 is repressed by c-myc and N-myc/Max complex in vitro (1). p53 induces the expression and nuclear translocation of NDRG1/Cap43 in response to DNA-damaging agents (4). In addition, NDRG1/ Cap43 is up-regulated by androgens (7) and various chemical agents including homocysteine and nickel compounds. The NDRG1/Cap43 expression is mostly observed in epithelial cells (8). NDRG1/Cap43 is also an essential factor for the maintenance of myelin sheaths in peripheral nerves (9). In particular, NDRG1/Cap43 is associated with the terminal differentiation of Schwann cells during nerve regeneration (10), and also with the differentiation of the kidney and brain (11). NDRG1/Cap43 is expressed at lower levels in tumor cells including prostate, breast and colorectal cancers than in normal tissue $(2,4,12)$, and can suppress metastasis by inducing cell differentiation and reversing the metastatic phenotype $(13,14)$. However, NDRG1/Cap43 is highly expressed in certain tumors, such as hepatocellular and colorectal carcinoma $(15,16)$. The NDRG1/Cap43 expression is positively associated with tumor angiogenesis in cervical adenocarcinoma (17). The positive or negative association between NDRG1/Cap43 and tumor angiogenesis depends upon tumor types and differentiation status (18). Although there is certain evidence that NDRG1/Cap43 is involved in cell differentiation and in the metastasis of cancer cells, little is known about the relationship between the NDRG1/Cap43 expression and the differentiation of mesenchymal cells.

Vitamin $\mathrm{D}_{3}$ can induce normal and leukemic hematopoietic cells to differentiate into cells with mature monocyte/ macrophage phenotypes, and can also induce a block in cell proliferation $(19,20)$. Vitamin $\mathrm{D}_{3}$ also affects cell differentiation in other cell types, including poorly differentiated 
keratinocytes (21), prostate cancer (22), and several breast cancer cells (23). Vitamin $\mathrm{D}_{3}$ is essential for calcium homeostasis and bone metabolism, and plays an important role in osteoblast phenotypes and in the differentiation of osteosarcoma cells. We have previously demonstrated that vitamin $\mathrm{D}_{3}$ induces cell differentiation by the transcriptional activation of p21 in p53-deficient osteosarcoma cells (24).

In the present study, we first examined whether NDRG1/ Cap43 could play a critical role in cell differentiation in osteosarcoma cells treated with vitamin $\mathrm{D}_{3}$. On the basis of our results, using small interfering RNA (siRNA) against human NDRG1/Cap43, we showed how NDRG1/Cap43 plays a role in cell differentiation and invasion by osteosarcoma cells.

\section{Materials and methods}

Cell culture and treatment. The human osteosarcoma cells, MG63, SaOS2 and U2OS, were used in this study. The cells were incubated in Dulbecco's modified Eagle's medium (DMEM) (Nissui, Japan) supplemented with $10 \%$ (v/v) fetal calf serum (FCS) (Bioserum, Victoria, Australia), 100 units $/ \mathrm{ml}$ penicillin, and $10 \mathrm{mg} / \mathrm{ml}$ streptomycin (Gibco/Life Technologies, Gettysburg, MD, USA).

In vitro cell proliferation assay. The cells were seeded into a 96-well culture plate $\left(1 \times 10^{4}\right.$ cells/well) and incubated for one day in a medium containing $0.1 \%$ FCS and indicated concentrations of vitamin $\mathrm{D}_{3}$. Cell proliferation was analyzed using a 3-(4,5-dimethylthiazol-2-yl) 2,5-diphenyltetrazolium bromide (MTT) assay. In order to estimate the influence of the NDRG1/Cap43 knockdown on cell proliferation in the U2OS cells, the cells $\left(5 \times 10^{3} /\right.$ well $)$ were plated in 6 -well plates in a medium supplemented with $10 \%$ serum. The cells were trypsinized and counted at $0,1,3$ and 5 days with a coulter counter (Beckman Coulter, Miami, FL).

Production of osteocalcin by osteosarcoma cells. For the measurement of osteocalcin, the cells were seeded in a 6-well culture plate and cultured to $70 \%$ confluence. The cells were washed three times with phosphate buffered saline (PBS) and incubated in serum-free medium for $24 \mathrm{~h}$ before the experiment. The cells were incubated for $36 \mathrm{~h}$ in a medium containing $0.1 \%$ FCS and indicated concentrations of vitamin $\mathrm{D}_{3}$. After the treatment, the medium was collected for the measurement of osteocalcin. This was performed using an Osteocalcin EIA kit (Takara, Japan).

NDRG1/Cap43 siRNA expression vector construction and establishment of NDRG1/Cap43 siRNA expressing cells. The pcDNA3_GFP_hU6 siRNA expression plasmid was kindly provided by Dr H. Izumi (University of Occupational and Environmental Health, Kitakyushu, Japan). In order to obtain the pcDNA3_GFP_hU6/Cap43 siRNA expression plasmid, the following double-stranded oligonucleotides were inserted into the pcDNA3_GFP_hU6 siRNA expression plasmid (Invitrogen, San Diego, CA) designed for BamHI and HindIII. The NDRG1/Cap43 siRNA oligonucleotides were as follows: sense, 5'-GATCCGCGTGAACCCTTGTGCGGAA TTCAAGAGATTCCGCACAAGGGTTCACGTTTT TTGGAAA-3'; and antisense, 5'-AGCTTTTCCAAAAAAC

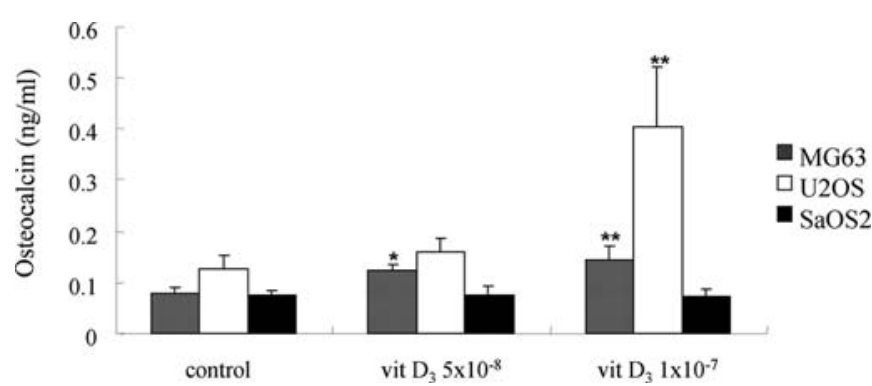

Figure 1. Effects of vitamin D3 on the production of osteocalcin in MG63, U2OS and SaOS2 cells. The cells were incubated in a medium containing $0.1 \%$ FCS with or without various concentrations of vitamin $\mathrm{D}_{3}\left(5 \times 10^{-8}\right.$ and $1 \times 10^{-7} \mathrm{~mol} / \mathrm{l}$ ) for $36 \mathrm{~h}$, and the conditioned medium was then collected. The measurement of osteocalcin was performed using an Osteocalcin EIA kit.

GTGAACCCTTGTGCGGAATCTCTTGAATTCCGCA CAAGGGTTCACGCG-3' (Invitrogen). The Cells were transfected with the pcDNA3_GFP_hU6/Cap43 siRNA expression plasmid (NDRG1/Cap43 siRNA) or the pcDNA3_GFP_hU6 siRNA expression plasmid (mock) using Lipofectamine 2000 (Invitrogen) following the manufacturer's protocol.

Western blot analysis. The cells were washed with ice-cold PBS, scraped from the plates, lysed, and harvested on ice in RIPA buffer supplemented with PMSF, protease inhibitor cock-tail and sodium orthovanadate (Santa Cruz Biotechnology, Inc., Santa Cruz, CA). The homogenates were clarified by centrifugation at $15,000 \times \mathrm{g}$ for $15 \mathrm{~min}$ at $4^{\circ} \mathrm{C}$, and the supernatant was collected. The proteins were separated using SDS-PAGE, loading $50 \mu \mathrm{g}$ aliquots of protein into each well, and transferred to Immobilon membranes (Millipore, Bedford, MA). After the transfer, the blots were incubated with blocking solution and probed with antibodies. The antibodies used were the following: Rabbit polyclonal antibody directed against NDRG1/Cap43, mouse monoclonal anti-p21 Waf1/ Cip1 (DCS60) antibody (Cell Signaling Technology, Beverly, MA), and mouse monoclonal anti-GAPDH antibody (SigmaAldrich, St. Louis, MO).

Statistical analysis. The significance of the differences in cell proliferation and the production of osteocalcin was estimated using the unpaired Student's t-test. The results were recognized as statistically significant at a probability value of $\mathrm{p}<0.05$.

\section{Results}

Effects of vitamin $D_{3}$ on cell differentiation, cell proliferation and NDRG1/Cap43 expression. We first examined whether vitamin $\mathrm{D}_{3}$ could induce cell differentiation in three osteosarcoma cell lines, MG63, U2OS and SaOS2. The secretion of osteocalcin is a representative biomarker of cell differentiation in osteoblasts. Osteocalcin was increased in a dosedependent manner by vitamin $\mathrm{D}_{3}$ in MG63 and U2OS, but not in SaOS2 (Fig. 1). There was an $\sim 2$-fold and 4-fold increase in the production of osteocalcin by vitamin $\mathrm{D}_{3}$ in MG63 and U2OS, respectively. In all three cell lines, cell proliferation was not affected after exposure to vitamin $D_{3}$ (Fig. 2). 


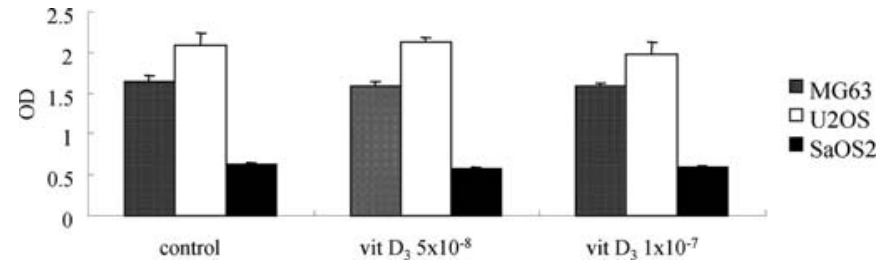

Figure 2. Effects of vitamin $\mathrm{D}_{3}$ on the cell proliferation in the MG63, U2OS and $\mathrm{SaOS} 2$ cell lines. The cells were incubated in a medium containing $0.1 \%$ FCS and various concentrations of vitamin $\mathrm{D}_{3}$ (none, $5 \times 10^{-8}$ and $\left.1 \times 10^{-7} \mathrm{~mol} / \mathrm{l}\right)$ for $48 \mathrm{~h}$, and the effects were analyzed using an MTT assay. "p $<0.05,{ }^{* *} \mathrm{p}<0.01$.

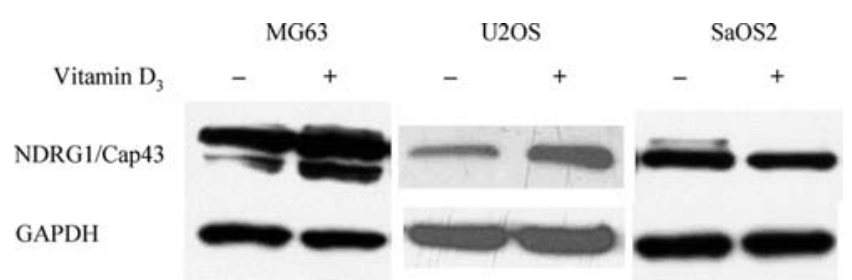

Figure 3. Effects of vitamin $\mathrm{D}_{3}$ on the expression of NDRG1/Cap43 in the MG63, U2OS and SaOS2 cell lines. Cells $\left(1 \times 10^{5}\right)$ were plated in 6-well plate and cultured in DMEM containing $0.1 \%$ FCS with or without vitamin $\mathrm{D}_{3}\left(1 \times 10^{-7} \mathrm{~mol} / \mathrm{l}\right)$ treatment. The expression of NDRG1/Cap43 was analyzed by Western blot analysis.

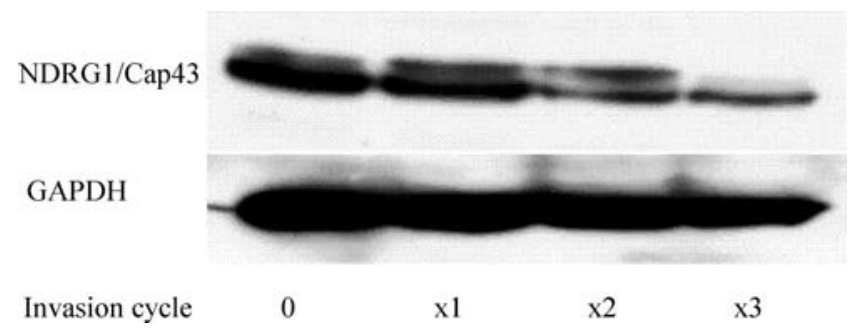

Figure 4. The decreased expression of NDRG1/Cap43 in highly invasive $\mathrm{SaOS} 2$ cells. The cells $\left(2 \times 10^{5}\right)$ were plated onto a coated filter in a Matrigel Invasion chamber. Migrated cells were collected after 24-h incubation, and were cultured under normal conditions. Subsequently, invasive cells were also incubated in the chamber for $24 \mathrm{~h}$. This procedure was performed three times. A fraction of the cells was collected and examined for NDRG1/ Cap43 expression via Western blot analysis.

Western blot analysis showed the expression of NDRG1/ Cap43 in MG63, SaOS2, and U2OS under exponentially growing conditions. The NDRG1/Cap43 expression was enhanced by vitamin $\mathrm{D}_{3}$ in MG63 and U2OS, but not in SaOS2 (Fig. 3).

NDRG1/Cap43 expression and invasiveness of osteosarcoma cells. The in vitro invasion assay was designed to test whether the invasive potential of the tumor cells had any correlation with the NDRG1/Cap43 expression and cell differentiation in a Matrigel-coated transwell chamber. SaOS2 was found to have the greatest invasive potential among the three cell lines (data not shown), and therefore we used $\mathrm{SaOS} 2$ for the invasion assay in this study. We isolated $\mathrm{SaOS} 2 \mathrm{x} 1, \mathrm{SaOS} 2 \mathrm{x} 2$ and $\mathrm{SaOS} 2 \mathrm{x} 3$ at each cycle of the
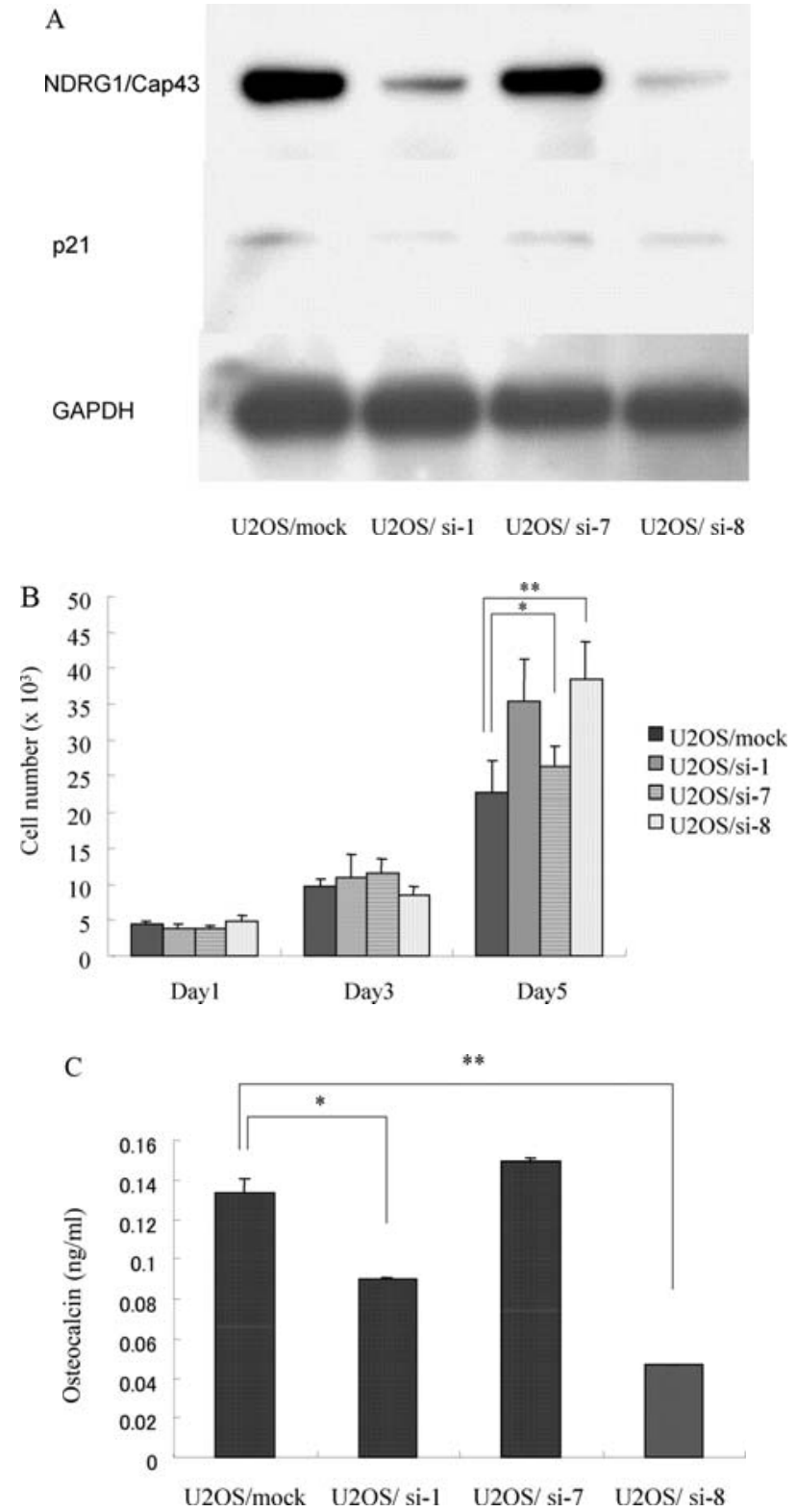

Figure 5. The effects of siRNA transfection on the expression of NDRG1/ Cap43 and p21 and the production of osteocalcin. Three human osteosarcoma cell lines constitutively expressing NDRG1/Cap43 siRNA, U2OS/si-1, U2OS/si-7 and U2OS/si-8, derived from human osteosarcoma U2OS cells, were established. The expression of the NDRG1/Cap43 protein was decreased in both the U2OS/si-1 and U2OS/si-8 cells compared to the $\mathrm{U} 2 \mathrm{OS} / \mathrm{si}-7$ and the parental cells. The p21 protein was also suppressed in these cell lines (A), resulting in an increase in cell proliferation in the U2OS/si-1 and U2OS/si-8 cells (B). The osteocalcin protein level was determined using EIA kits. The production of osteocalcin was significantly suppressed by the decreased expression of NDRG1/Cap43 in U2OS/si-1 and U2OS/si-8 cells (C). Each value represents the mean concentration of osteocalcin \pm SD. ${ }^{*} \mathrm{p}<0.05,{ }^{* *} \mathrm{p}<0.01$.

invasion assay, and compared the expression levels of NDRG1/Cap43 in those cell lines. There was a markedly decreasing expression of NDRG1/Cap43 during the acquirement of the invasive potential by the $\mathrm{SaOS} 2$ cells. NDRG1/ Cap43 often showed two major bands: The upper band was phosphorylated and the lower band was relatively phosphorylated, as the treatment with phosphatase resulted in the almost complete disappearance of the upper band of NDRG1/ Cap43 (unpublished data). There was a much lower 
expression of the phosphorylated band of NDRG1/Cap43 in $\mathrm{SaOS} 2 \mathrm{x} 2$ and $\mathrm{SaOS} 2 \mathrm{x} 3$ compared to $\mathrm{SaOS} 2$ and $\mathrm{SaOS} 2 \mathrm{x} 1$ (Fig. 4).

Cell differentiation and NDRG1/Cap43 knockdown. In order to verify the relationship between the NDRG1/Cap43 expression and cell differentiation, we established two human osteosarcoma cell lines constitutively expressing NDRG1/ Cap43 siRNA, U2OS/si-1 and U2OS/si-8, derived from human osteosarcoma U2OS cells. The expression of the NDRG1/Cap43 protein was markedly down-regulated in both the U2OS/si-1 and U2OS/si-8 cells compared to their parental counterpart, U2OS/mock (Fig. 5A). The U2OS/mock cells showed relatively the same levels of NDRG1/Cap43 as the U2OS cells (data not shown). Furthermore, the expression of the p21 protein was also down-regulated in both the NDRG1/Cap43 knockdown cell lines (Fig. 5A). Cell proliferation rates were found to be significantly increased in U2OS/ si-1 and U2OS/si-8, compared to U2OS/mock (Fig. 5B).

We also compared the differentiation between the NDRG1/Cap43-knockdown cell lines and their parental counterpart. As shown in Fig. 5C, the production of osteocalcin was significantly down-regulated by the NDRG1/ Cap43 knockdown in U2OS/si-1 $(\mathrm{p}<0.05)$ and U2Os/si-8 $(\mathrm{p}<0.01)$, indicating a close correlation between cell differentiation and NDRG1/Cap43 in osteosarcoma cells.

\section{Discussion}

In this study, we demonstrated that the NDRG1/Cap43 expression is increased concomitantly with the promotion of cell differentiation by vitamin $\mathrm{D}_{3}$ in the two osteosarcoma cell lines, MG63 and U2OS. However, the NDRG1/Cap43 expression was not affected by vitamin $\mathrm{D}_{3}$, which failed to induce cell differentiation in SaOS2. Mahonen et al, have reported the differences between MG63 and SaOS2 in the response of type I collagen synthesis to vitamin $\mathrm{D}_{3}(25)$. Zehenter et al, have also reported that BMP-2 increases Sox 9 mRNA levels in MG63 but not in other osteosarcoma cell lines (26). The failure in the expression of both NDRG1 and osteocalcin in the SaOS2 cells could be due to the loss of function of the vitamin $\mathrm{D}_{3}$ signaling, pathway including its cognate receptor. However, the precise mechanisms causing these three cell lines to show different responses to vitamin $\mathrm{D}_{3}$, is not clear.

We have previously reported that the gelatinolytic activity by matrix metalloproteinase- 9 and the invasive ability during the Matrigel invasion, are both decreased in pancreatic cancer cell lines with a high NDRG1/Cap43 expression (27). In this study, during the acquirement of the invasion activity by $\mathrm{SaOS} 2$, the expression of the NDRG1/Cap43 protein was markedly decreased, suggesting a close correlation between NDRG1/Cap43 and the invasive potential of osteosarcoma cells. However, Matrigel did not affect the invasion of MG63 at all (data not shown). This response was possibly influenced by the differences in the differentiation state of these cells: MG63 cells are considered to be early osteoblast-like cells, whereas SaOS2 cells are not as well differentiated as the MG63 cells. Furthermore, the upper band, corresponding to the phosphorylated NDRG1/Cap43, was markedly decreased in the $\mathrm{SaOS} 2 \mathrm{x} 2$ and $\mathrm{SaOS} 2 \mathrm{x} 3$ cells. The phosphorylation of NDRG1/Cap43 is mediated through SGK1 and GSK3, essential Ser/Thr kinase family proteins (28). Further study is required in order to determine how the phosphorylation by these enzymes could be modified during the acquisition of invasiveness of osteosarcoma cells in Matrigel.

Kurdistani et al, have previously reported that the NDRG1/Cap43 expression was induced by wild-type-p53, and demonstrated a parallel increase in the expression of NDRG1/Cap43 and p21, and the CdK inhibitor in SaOS2 cells (4). Based on these findings, we established two osteosarcoma cell lines, derived from U2OS, with a decreased NDRG1/Cap43 expression, in order to examine the relationship between the NDRG1/Cap43 and p21 expression and cell differentiation. p21 is involved in cell differentiation via the p53-independent pathway in various cell lineages including osteosarcoma (29-31). In U2OS/si-1 and U2OS/si-8 with a decreased NDRG1/Cap43 expression, the p21 expression was down-regulated, resulting in enhanced cell proliferation compared to the parental cells. The production of osteocalcin was suppressed by the NDRG1/Cap43 knockdown. On the whole, our present study strongly suggests that NDRG1/ Cap43 plays a pivotal role in the cell differentiation of osteosarcoma cells in close association with p21.

In conclusion, we demonstrated that NDRG1/Cap43 contributed to the regulation of cell differentiation as well as cell proliferation by osteosarcoma cells. Furthermore, NDRG1/Cap43 also affects cell motility and invasion by osteosarcoma cells. NDRG1/Cap43 could be useful as a molecular target for the development of novel therapeutics against osteosarcoma.

\section{Acknowledgements}

We would like to thank Kazuhiro Yoshida and Hitomi Wakita for their technical assistance. This study was partly supported by a Grant-in-Aid for Scientific Research (C) (no. 18591646) from the Japanese Ministry of Education, Science, Sports and culture, and partly by the 21 st Century COE Program for Medical Science.

\section{References}

1. Shimono A, Okuda T and Kondoh H: N-myc-dependent repression of NDR1, a gene identified by direct subtraction of whole mouse embryo cDNAs between wild type and $\mathrm{N}$-myc mutant. Mech Dev 83: 39-52, 1999.

2. Van Belzen N, Dinjens WN, Diesveld MP, et al: A novel gene which is up-regulated during colon epithelial cell differentiation and down-regulated in colorectal neoplasms. Lab Invest 77: 85-92, 1997.

3. Kokame K, Kato H and Miyata T: Homocysteine-respondent genes in vascular endothelial cells identified by differential display analysis. GRP78/BIP and novel genes. J Biol Chem 271: 29659-29665, 1996.

4. Kurdistani SK, Arizti P, Reimer CL, Sugrue MM, Aaronson SA and Lee SW: Inhibition of tumor cell growth by RTP/RIT42 and its responsiveness to p53 and DNA damage. Cancer Res 58: 4439-4444, 1998.

5. Zhou RH, Kokame K, Tsukamoto Y, Yutani C, Kato H and Miyata T: Characterization of the human NDRG gene family: A newly identified member, NDRG4, is specifically expressed in brain and heart. Genomics 73: 86-97, 2001.

6. Agarwala KL, Kokame K, Kato H and Miyata T: Phosphorylation of RTP, an ER stress-responsive cytoplasmic protein. Biochem Biophys Res Commun 272: 641-647, 2000. 
7. Lin TM and Chang C: Cloning and characterization of TDD5, an androgen target gene that is differentially repressed by testosterone and dihydrotestosterone. Proc Natl Acad Sci USA 94: 4988-4993, 1997.

8. Lachat P, Shaw P, Gebhard S, van Belzen N, Chaubert P and Bosman FT: Expression of NDRG1, a differentiation-related gene, in human tissues. Histochem Cell Biol 118: 399-408, 2002 .

9. Okuda T, Higashi Y, Kokame K, Tanaka C, Kondoh H and Miyata T: NDRG1-deficient mice exhibit a progressive demyelinating disorder of peripheral nerves. Mol Cell Biol 24: 3949-3956, 2004.

10. Hirata K, Masuda K, Morikawa W, et al: N-myc downstreamregulated gene 1 expression in injured sciatic nerves. Glia 47: 325-334, 2004

11. Wakisaka Y, Furuta A, Masuda K, Morikawa W, Kuwano M and Iwaki T: Cellular distribution of ndrg1 protein in the rat kidney and brain during normal postnatal development. J Histochem Cytochem 51: 1515-1525, 2003.

12. Nishie A, Masuda K, Otsubo M, et al: High expression of the Cap43 gene in infiltrating macrophages of human renal cell carcinomas. Clin Cancer Res 7: 2145-2151, 2001.

13. Guan RJ, Ford HL, Fu Y, Li Y, Shaw LM and Pardee AB: DRG-1 as a differentiation-related, putative metastatic suppressor gene in human colon cancer. Cancer Res 60: 749755,2000

14. Bandyopadhyay S, Wang Y, Zhan R, et al: The tumor metastasis suppressor gene DRG-1 down-regulates the expression of activating transcription factor 3 in prostate cancer. Cancer Res 66: 11983-11990, 2006.

15. Chua MS, Sun H, Cheung ST, et al: Overexpression of NDRG1 is an indicator of poor prognosis in hepatocellular carcinoma. Mod Pathol 20: 76-83, 2007.

16. Wang Z, Wang F, Wang WQ, et al: Correlation of n-myc downstream-regulated gene 1 overexpression with progressive growth of colorectal neoplasm. World J Gastroenterol 10: 550$554,2004$.

17. Nishio S, Ushijima K, Tsuda N, et al: Cap43/NDRG1/DRG-1 is a molecular target for angiogenesis and a prognostic indicator in cervical adenocarcinoma. Cancer Lett 264: 36-43, 2008.

18. Hosoi F, Izumi H, Kawahara A, et al: N-myc downstream regulated gene 1/Cap43 suppresses tumor growth and angiogenesis of pancreatic cancer through attenuation of inhibitor of kappaB kinase beta expression. Cancer Res 69: 4983-4991, 2009.

19. Bar-Shavit Z, Teitelbaum SL, Reitsma P, et al: Induction of monocytic differentiation and bone resorption by 1,25-dihydroxyvitamin D3. Proc Natl Acad Sci USA 80: 5907-5911, 1983.
20. Munker R, Norman A and Koeffler HP: Vitamin D compounds. Effect on clonal proliferation and differentiation of human myeloid cells. J Clin Invest 78: 424-430, 1986.

21. Bikle DD and Pillai S: Vitamin D, calcium, and epidermal differentiation. Endocr Rev 14: 3-19, 1993.

22. Esquenet M, Swinnen JV, Heyns W and Verhoeven G: Control of INCAP proliferation and differentiation: Actions and interactions of androgens, 1alpha,25-dihydroxycholecalciferol, alltrans retinoic acid, 9-cis retinoic acid, and phenylacetate. Prostate 28: 182-194, 1996.

23. Elstner E, Linker-Israeli M, Said J, et al: 20-epi-vitamin D3 analogues: A novel class of potent inhibitors of proliferation and inducers of differentiation of human breast cancer cell lines. Cancer Res 55: 2822-2830, 1995.

24. Zenmyo M, Komiya S, Hamada T, et al: Transcriptional activation of $\mathrm{p} 21$ by vitamin $\mathrm{D}(3)$ or vitamin $\mathrm{K}(2)$ leads to differentiation of p53-deficient MG-63 osteosarcoma cells. Hum Pathol 32: 410-416, 2001

25. Mahonen A, Jukkola A, Risteli L, Risteli J and Maenpaa PH: Type I procollagen synthesis is regulated by steroids and related hormones in human osteosarcoma cells. J Cell Biochem 68: 151-163, 1998 .

26. Zehentner BK, Dony $\mathrm{C}$ and Burtscher $\mathrm{H}$ : The transcription factor Sox9 is involved in BMP-2 signaling. J Bone Miner Res 14: 1734-1741, 1999

27. Maruyama Y, Ono M, Kawahara A, et al: Tumor growth suppression in pancreatic cancer by a putative metastasis suppressor gene Cap43/NDRG1/DRG-1 through modulation of angiogenesis. Cancer Res 66: 6233-6242, 2006.

28. Murray JT, Campbell DG, Morrice N, et al: Exploitation of kestrel to identify NDRG family members as physiological substrates for SGK1 and GSK3. Biochem J 384: 477-488, 2004.

29. Jiang H, Lin J, Su ZZ, Collart FR, Huberman E and Fisher PB: Induction of differentiation in human promyelocytic HL-60 leukemia cells activates p21, waf1/cip 1 , expression in the absence of p53. Oncogene 9: 3397-3406, 1994.

30. Halevy O, Novitch BG, Spicer DB, et al: Correlation of terminal cell cycle arrest of skeletal muscle with induction of p21 by MYOD. Science 267: 1018-1021, 1995.

31. Schwaller J, Koeffler HP, Niklaus G, et al: Posttranscriptional stabilization underlies p53-independent induction of p21Waf1/ Cip1/SDI1 in differentiating human leukemic cells. J Clin Invest 95: 973-979, 1995. 